\title{
Direct Evidence for an Intrinsic Square Vortex Lattice in the Overdoped High- $T_{c}$ Superconductor $\mathrm{La}_{1.83} \mathrm{Sr}_{0.17} \mathrm{CuO}_{4+\delta}$
}

\author{
R. Gilardi, ${ }_{1}^{1}$ J. Mesot, ${ }^{1}$ A. Drew, ${ }^{2}$ U. Divakar, ${ }^{2}$ S. L. Lee, ${ }^{2}$ E. M. Forgan, ${ }^{3}$ O. Zaharko, ${ }^{1}$ K. Conder, ${ }^{1}$ V. K. Aswal, ${ }^{4}$ \\ C. D. Dewhurst, ${ }^{5}$ R. Cubitt, ${ }^{5}$ N. Momono, ${ }^{6}$ and M. Oda ${ }^{6}$ \\ ${ }^{1}$ Laboratory for Neutron Scattering, ETH Zurich and PSI Villigen, CH-5232 Villigen PSI, Switzerland \\ ${ }^{2}$ School of Physics and Astronomy, University of St. Andrews, Fife, KY16 9SS, United Kingdom \\ ${ }^{3}$ School of Physics and Astronomy, University of Birmingham, Birmingham B15 2TT, United Kingdom \\ ${ }^{4}$ Spallation Neutron Source Division, PSI Villigen, CH-5232 Villigen PSI, Switzerland \\ ${ }^{5}$ Institut Laue-Langevin, BP 156, F-38042 Grenoble, France \\ ${ }^{6}$ Department of Physics, Hokkaido University, Sapporo 060-0810, Japan
}

(Received 20 December 2001; published 10 May 2002)

\begin{abstract}
We report here the first direct observations of a well ordered vortex lattice in the bulk of a $\mathrm{La}_{2-x} \mathrm{Sr}_{x} \mathrm{CuO}_{4+\delta}$ single crystal (slightly overdoped, $x=0.17$ ). Our small angle neutron scattering investigation of the mixed phase reveals a crossover from triangular to square coordination with increasing magnetic field. The existence of an intrinsic square vortex lattice has never been observed in high-temperature superconductors and is indicative of the coupling of the vortex lattice to a source of anisotropy, such as those provided by a $d$-wave order parameter or the presence of stripes.
\end{abstract}

DOI: $10.1103 /$ PhysRevLett.88.217003

PACS numbers: 74.60.Ge, 61.12.Ex, 74.72.Dn

Apart from the unusual electronic and magnetic behavior of the cuprate high-temperature superconductors (HTSC), experiments reveal a tremendously rich variety of mesoscopic phenomena associated with the flux vortices in the mixed state [1]. Because of their two-dimensional electronic structure, the HTSC are highly anisotropic. The anisotropy is characterized by the ratio $\gamma=\lambda_{\perp} / \lambda_{\|}$, where $\lambda_{\perp}, \lambda_{\|}$are the superconducting penetration depths for currents flowing perpendicular and parallel to the two-dimensional $\mathrm{CuO}_{2}$ planes. In $\mathrm{La}_{2-x} \mathrm{Sr}_{x} \mathrm{CuO}_{4+\delta}$ (La214) the degree of anisotropy ( $\gamma \approx 20$ for $x=0.15$ ) lies between that of the $\mathrm{YBa}_{2} \mathrm{Cu}_{3} \mathrm{O}_{x} \quad(\mathrm{Y} 123)$ and $\mathrm{Bi}_{2} \mathrm{Sr}_{2} \mathrm{CaCu}_{2} \mathrm{O}_{8+x}$ (Bi2212) materials [2]. The cuprates are also extreme type-II superconductors, indicated by the high value of the Ginzburg-Landau parameter $\kappa=\lambda / \xi$, where $\xi$ is the superconducting coherence length. In HTSC the combination of high transition temperature $T_{c}$, high $\gamma$ and high $\kappa$ leads to exotic vortex behavior, such as the phenomenon of vortex lattice (VL) melting [1]. For a conventional (isotropic) pairing mechanism such anisotropic conduction properties can lead to distortions of the vortex lattice as the applied field is tilted towards the $\mathrm{CuO}_{2}$ planes, but the local coordination remains sixfold [3-6].

From the magnetic point of view the single-layer (one $\mathrm{CuO}_{2}$ sheet per unit cell) La214 compounds appear to be rather different from their two-layered Y123 and Bi2212 counterparts. La214 is characterized by the presence of incommensurate spin excitations [7] located in the vicinity of the antiferromagnetic wave vector $(\pi, \pi)$ of the undoped parent compound and at low temperature a spin gap develops in the spectrum. While such excitations could originate from coherence effects in the superconducting state [8] it has also been proposed that they could indicate the presence of dynamical stripes [9]. It is still unclear to what extent the presence of dynamical stripes and/or antiferromagnetic fluctuations affects the vortex state. Recently, inelastic neutron scattering experiments in a magnetic field have revealed subgap excitations which have been attributed to the existence of antiferromagnetic excitations in the vortex core [10]. It was also shown that the opening of the spin gap is correlated to the melting line of the VL rather than $T_{c}[10,11]$. These results indicate that a subtle interplay may indeed exist between the unusual microscopic and mesoscopic properties of the La214 compounds.

Our experiments were performed on the small angle neutron scattering (SANS) instruments of both the Paul Scherrer Institute (PSI) [12], Switzerland, and of the Institut Laue Langevin (D22), Grenoble, France, with a neutron velocity selector of $10 \%$ full width at half maximum, to produce a flux of neutrons with a mean wavelength $\lambda_{0}$ between 6 and $15 \AA$. The incident neutrons were collimated over a distance of $18 \mathrm{~m}$ before reaching the sample. A $96 \times 96 \mathrm{~cm}^{2}$ multidetector [13] at a distance of $18 \mathrm{~m}$ was used to detect the scattered neutrons.

The sample [14] was a single crystal cylinder of length $20 \mathrm{~mm}$, diameter $6 \mathrm{~mm}$ with the $c$ axis perpendicular to the cylinder. One of the $\{1,1\}$ in-plane axes, which correspond to the directions of the $\mathrm{Cu}-\mathrm{O}$ bonds of the $\mathrm{CuO}_{2}$ planes, lay roughly along the cylinder axis. The crystal had composition $\mathrm{La}_{1.83} \mathrm{Sr}_{0.17} \mathrm{CuO}_{4}\left(T_{c}=37 \mathrm{~K}\right)$. The width of the superconducting transition, measured by ac susceptibility, was quite small $\left(\Delta T_{c}=1.3 \mathrm{~K}\right.$, as defined by the $10 \%-90 \%$ criterion), indicating the high sample quality. It was mounted in a cryostat in a magnetic field of $0-1.2 \mathrm{~T}$ applied parallel to the incident beam.

For the initial measurements, the $c$ axis was oriented along the field direction. In a SANS experiment a conventional VL should give rise to Bragg reflections at reciprocal 
lattice points $q_{h k}$. The intensity of a single $(h, k)$ reflection is given by $I_{h k} \propto\left|F_{h k}\right|^{2} / q_{h k}$, where $F_{h k}$ is a spatial Fourier component of the field profile in the vortex lattice. For the case of a high- $\kappa$ superconductor such as La214 with the field perpendicular to the superconducting planes, in the London limit (fields well below the upper critical field) the form factor is $F_{h k}=B /\left[1+\left(q_{h k} \lambda_{\|}\right)^{2}\right]$, where $B$ is the average magnetic induction. This expression may easily be modified to account for an anisotropic system [3]. For $B>\mu_{0} H_{c 1}\left(H_{c 1}\right.$ is the lower critical field) the intensity of a first order reflection reduces to $I_{10} \propto d_{10} \Phi_{0}^{2} / \lambda_{\|}^{4}$, where $q_{10}=2 \pi / d_{10}$ and $\Phi_{0}$ is the flux quantum $h / 2 e$. In La214, the London penetration depth is similar to that measured in Bi2212 [15]. Although SANS has been used extensively to measure VL structure in both the Bi2212 [16] and Y123 systems [4-6], to date the La214 family remains virtually unexplored [17].

Figure 1 shows the difference of D22 data taken after cooling from $T=40 \mathrm{~K}>T_{c}$ to $T=1.5 \mathrm{~K}$ at $0.1 \mathrm{~T}$ and a background taken at $T=40 \mathrm{~K}$. Figure 2 shows similar PSI data at $0.8 \mathrm{~T}$ with a background taken at low temperature in zero field. These subtractions are a clean way to remove the large background signal arising from small angle defect scattering from the crystal. Because of the large mosaic of the vortex lattice, several spots could be observed simultaneously. We could furthermore rotate the cryomagnet and sample about the vertical and horizontal axes, allowing us to collect the integrated magnetic intensity as we rocked the Bragg spots through the Ewald sphere. Figure 3 a shows the tangential average of the neutron signal, as a function of the modulus of the wave vector q. As expected, the position (in reciprocal space) of the peak maximum changes with the field, thus clearly establishing the VL origin of the neutron signal.

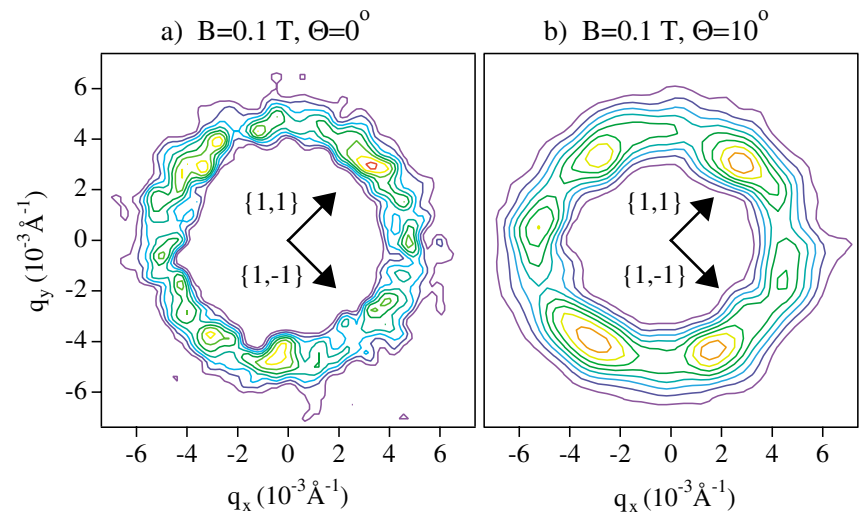

FIG. 1 (color). SANS diffraction patterns taken at $T=1.5 \mathrm{~K}$, after field cooling from $40 \mathrm{~K}$ in $B=0.1 \mathrm{~T}$. A background taken at $T=40 \mathrm{~K}$ has been subtracted. One of the $\{1,1\}$ in-plane axes is aligned at $45^{\circ}$ to the horizontal axis in the plane perpendicular to the field. In (a) the $c$ axis lies along the field direction and the field was kept constant during cooling (high-resolution mode: $\lambda_{0}=14.5 \AA$ ). In (b) the $c$ axis has been rotated $10^{\circ}$ (about the vertical axis) away from the field direction, and while cooling the field was oscillated about its mean value, $\lambda_{0}=8 \AA$.
We now return to the lowest-field data $(B=0.1 \mathrm{~T})$ shown in Fig. 1. When the $c$ axis lies along the field direction and the field is kept constant during cooling, we observe a large number of spots distributed around a ring (Fig. 1a, high-resolution mode: $\lambda_{0}=14.5 \AA$ ). Since the value of the wave vector obtained from the tangential average is close to the one expected for a triangular lattice (see Fig. 3b), the pattern most likely consists of a superposition of diffraction from various domain orientations of triangular coordination. In order to confirm this triangular coordination, it is necessary to reduce the high degree of degeneracy apparent in Fig. 1a. This was achieved by rotating the $c$ axis (about the vertical axis) away from the field direction by a sufficiently large angle $\Theta$. Further improvement of the mosaic spread of the VL was obtained by oscillating the field around its mean value while cooling. The results of these procedures are shown in Fig. 1b $\left(\Theta=10^{\circ}, \lambda_{0}=8 \AA\right)$, where a triangular pattern can be observed. Our measurements shown in Fig. 1 are very reminiscent of what has been seen in untwinned Y123 [5] crystals. In that system, a small amount of residual twin planes appears to control the orientation of the VL, giving rise to a high level of degeneracy. As the $c$ axis is rotated away from the field direction, the triangular symmetry becomes evident.

As the field is increased from 0.1 to $0.8 \mathrm{~T}$ a completely different pattern emerges, with all of the magnetic scattering concentrated in four intense spots appearing along the $\{1,1\}$ directions, forming a perfect square (Fig. 2a). To establish the significance of this fourfold diffraction pattern at higher field we must first take account of the influence of the twin planes. While the alignment of such patterns has previously been attributed to the influence of an anisotropic

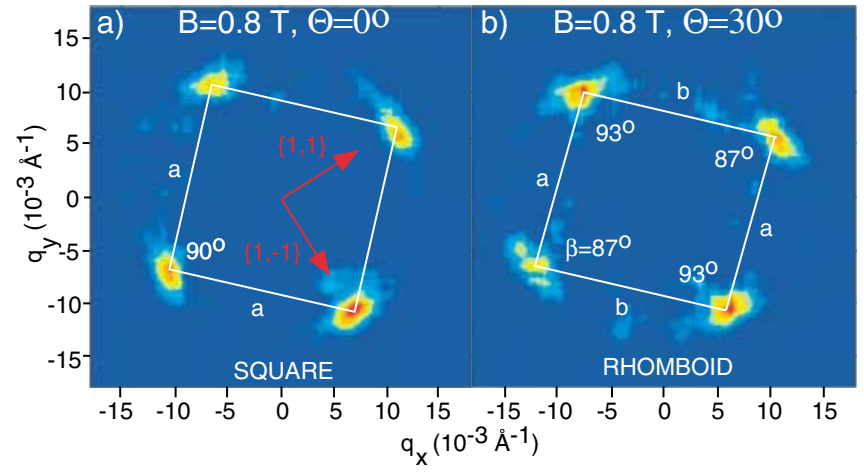

FIG. 2 (color). SANS diffraction patterns obtained by subtracting the zero-field data at $5 \mathrm{~K}$ from the data taken at $5 \mathrm{~K}$ for a field $B=0.8 \mathrm{~T}$ applied parallel to the beam, $\lambda_{0}=8 \AA$. One of the $\{1,1\}$ in-plane axes is aligned at $32^{\circ}$ to the horizontal axis in the plane perpendicular to the field. The $c$ axis of the sample is either (a) parallel to the field or (b) rotated $30^{\circ}$ away from it. The result of the two-dimensional fits of the diffraction patterns are shown in (a) by the white square and in (b) by the white rhomboid which illustrates the distortion induced by the rotation of the $c$ axis away from the direction of the applied magnetic field. 

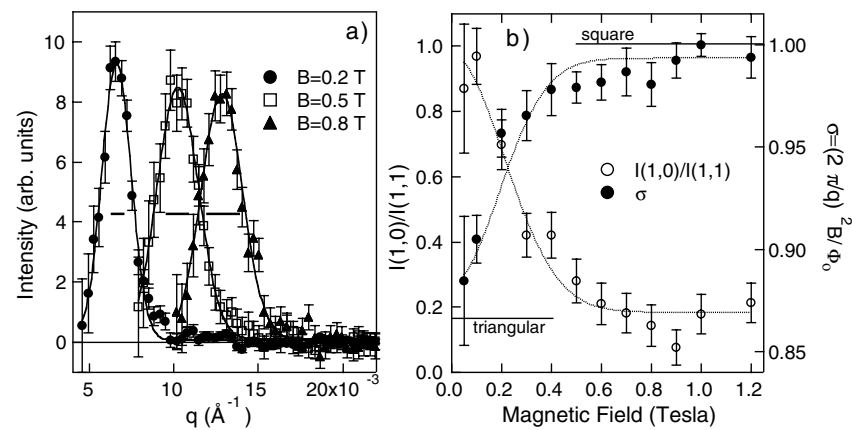

FIG. 3. (a) Tangential average of the neutron signal for $B=$ $0.2 \mathrm{~T}$ (circles), $0.5 \mathrm{~T}$ (squares), and $0.8 \mathrm{~T}$ (triangles) at $T=$ $5 \mathrm{~K}$. The intensities have been rescaled to allow a common vertical axis. The shift in $q$ illustrates the expected dependence of the vortex lattice spacing on increasing magnetic field. The instrumental resolution (FWHM) is given by the horizontal segments. (b) The black circles show the field dependence of $\sigma=(2 \pi / q)^{2} B / \Phi_{0}$ at $T=1.5 \mathrm{~K}$. The horizontal lines represent the expected values of $\sigma$ for triangular and square lattices. The open circles show the intensity ratio of sectors containing the $\{1,0\}$ and $\{1,1\}$ directions.

vortex core in Y123 [18], more recent measurements on untwinned Y123 crystals [5] have confirmed earlier suggestions [4,19] that these results in twinned crystals are actually explained in terms of strong alignment of the triangular VL mainly due to twin-plane pinning. Thus in Y123 when the field is roughly perpendicular to the $a b$ planes, an intrinsic triangular coordination may give rise to a predominantly fourfold diffraction pattern. This conclusion is also inferred in twinned Y123 by rotating the field away from the $c$ axis so that the influence of the twins is severely reduced, resulting in the recovery of a triangular coordination [5]. In Y123 for small tilt angles $\Theta<\Theta_{c} \approx 5^{\circ}$ a fraction of vortices remains pinned to the $c$ axis, since the vortices bend in order to lie for part of their length within the planar defects $[1,4]$. For larger angles this is no longer energetically favorable and the vortices lie along the direction of the applied field [6]. Estimates of this critical angle $\Theta_{c}$ for La214 would not suggest a significantly different behavior from Y123, given the slightly increased values for $\lambda$ and $\gamma$, although the precise value is difficult to predict [1] and falls with increasing field. Our measurements at low field (see Fig. 1) indicate that for our La214 compound $\Theta_{c}$ is lower than $10^{\circ}$. Hence, for data taken at high field, we can be sure that $\Theta=30^{\circ}$ exceeds the critical angle. We see in Fig. 2b that a four-spot pattern is retained at this angle. Thus it is extremely unlikely that this pattern arises in La214 from pinning distortions due to the presence of twin boundaries, and these data represent the first such observations of an intrinsically square VL in a cuprate HTSC. For completeness we note that for $\Theta=30^{\circ}$ there exists a slight distortion of the square pattern which is in accord with expectations for fields tilted away from the $c$ axis. Because of the approximately uniaxial superconducting anisotropy, the tilting causes an in- crease of the penetration depth of the vortex currents along one direction, distorting the vortex shape and hence the vortex lattice. In the London approach for anisotropic uniaxial superconductors [20] a rhomboid is expected. The expected values for the ratio $\epsilon=a / b$ between the sides ( $a$ and $b$ ) of the rhomboid and for the internal angle $\beta$ are $\epsilon=0.88, \beta=86.4^{\circ}$. A two-dimensional fit of the neutron data yields $\epsilon=0.90(3)$ and $\beta=87.3^{\circ}\left(1.7^{\circ}\right)$, which agree well with the theoretical values. Furthermore, the scattered intensity changes roughly as $\cos ^{2}(\Theta)$ as expected from the changes in the penetration depth. This indicates that all of the vortices lie along the field direction and not along the $c$ axis; that is, they are not pinned within the twin planes. Further contributory evidence for a square coordination comes from the positions of the Bragg spots in reciprocal space. The relationship between the magnetic field $B$ and the magnitude $q$ of the wave vector depends on a structure-dependent quantity $\sigma=(2 \pi / q)^{2} B / \Phi_{0}$, where $\sigma$ is equal to $\sqrt{3} / 2$ or 1 for triangular and square lattices, respectively. At high magnetic field, the experimental values of $\sigma$ are as expected for a square lattice, while at low field they are consistent with that of a triangular lattice (see Fig. 3b). An alternative way to quantify the triangular to square transition consists in monitoring the intensity ratio of sectors $\left( \pm 15^{\circ}\right)$ containing the $\{1,0\}$ - and $\{1,1\}$-type directions. As shown in Fig. $3 \mathrm{~b}$ this intensity decreases steadily from 1 at $B=0.1 \mathrm{~T}$ to about 0.2 at $B=0.4 \mathrm{~T}$ and then remains constant.

The experimental evidence thus indicates that an intrinsic square vortex lattice exists at fields $0.5 \mathrm{~T}$ and larger. We now discuss the possible origins of this fourfold coordination. Isotropic London or Ginzburg-Landau (GL) theories predict a triangular VL in high- $\kappa$ systems such as the cuprates. This is also true for modified theories which take into account the anisotropy of layered materials such as the HTSC. VL of square coordination has, however, been observed in noncuprate superconductors and may occur for a number of reasons. In low- $\kappa$ materials nonlocal electrodynamic effects are expected to be more significant due to stronger interactions between the cores of neighboring vortices. This may give rise to a VL of square coordination, although at low magnetic induction (large vortex separation) or close to $T_{c}$ (reduced influence of nonlocal effects) the VL tends to a triangular coordination. In combination with anisotropic electronic structure, this explains the recent observations of triangular to square transitions in the nickel boron-carbide superconductors [21]. A square VL can also arise in extended GL theories which allow for the existence of more than one order parameter, which may account for the robustness of the square VL in the candidate $p$-wave system $\mathrm{Sr}_{2} \mathrm{RuO}_{4}$ [22]. A fourfold symmetry could also result from the anisotropic ( $d$-wave) nature of the superconducting gap [23-26] via the increasing importance of the anisotropic vortex cores at high field. The onset of such effects might be expected to occur at fields a factor of order 10 lower than in Y123 $\left(T_{c}^{\max }=93 \mathrm{~K}\right)$, due 
to the smaller gap in $\mathrm{La} 214\left(T_{c}^{\max }=38.5 \mathrm{~K}\right)$. The experimental search for such a transition has been complicated in Y123 by the influence of twin-plane pinning discussed above. To date there are no reports on either twinned or untwinned Y123 for such an intrinsic change of coordination. The only evidence for a structural change as a function of field is a reorientation of the triangular lattice [5] starting at fields of around $3 \mathrm{~T}$. In Bi2212 the vortex lattice cannot be observed using SANS at high fields, due to the disorder induced by point pinning [16]. It is interesting to notice that the early VL calculations based on $d$-wave gap functions $[23,24]$ predict the square lattice to be oriented along the $\mathrm{Cu}-\mathrm{O}-\mathrm{Cu}$ bonds $(\{1,1\}$ direction in La214), which is exactly what our experimental data reveal. However, more recent VL calculations [25,26] favor a square lattice tilted by $45^{\circ}$ from the $\mathrm{Cu}-\mathrm{O}-\mathrm{Cu}$ bonds. Our results provide an important stimulus to resolve these theoretical discrepancies. In principle, other sources of anisotropy, such as those involving the presence of dynamical stripes [27] or charge-density waves [28], could lead to the formation of a square vortex lattice. However, the plausibility of this explanation is as yet unclear, since our measurements were performed in the overdoped regime of the HTSC where a homogeneous Fermi liquid description of the electronic properties of these materials is currently believed to be adequate.

In conclusion, we have used SANS to make the first clear observation of the vortex lattice in La214 and obtained strong evidence for a field-induced crossover from a triangular to a square lattice. This may reflect the increasing importance of the anisotropic vortex cores in this $d$-wave superconductor, or coupling to other sources of anisotropy such as those provided by charge/stripe fluctuations. It remains a challenge to corroborate detailed SANS measurements with other microscopic data in order to explain the origin of this exotic vortex behavior.

We thank T. Konter, J. Kohlbrecher, A. Bollhalder, P. Rasmussen, and N. Schlumpf for the technical assistance. This work was supported by the Swiss National Science Foundation, the Engineering and Physical Sciences Research Council of U.K., and the Ministry of Education and Science of Japan.
[1] For a review, see G. Blatter et al., Rev. Mod. Phys. 66, 1125 (1994).

[2] T. Sasagawa et al., Phys. Rev. B 61, 1610 (2000).

[3] S. L. Thiemann et al., Phys. Rev. B 39, 11406 (1989).

[4] M. Yethiraj et al., Phys. Rev. Lett. 70, 857 (1993).

[5] S. T. Johnson et al., Phys. Rev. Lett. 82, 2792 (1999).

[6] M. Yethiraj et al., Phys. Rev. Lett. 71, 3019 (1993).

[7] T. E. Mason, G. Aeppli, and H. A. Mook, Phys. Rev. Lett. 68, 1414 (1992); K. Yamada et al., Phys. Rev. Lett. 75, 1626 (1995).

[8] N. Bulut et al., Phys. Rev. Lett. 64, 2723 (1990); Y. Zha, K. Levin, and Qimiano Si, Phys. Rev. B 47, 9124 (1993); M. R. Norman, Phys. Rev. B 61, 14751 (2000).

[9] J. M. Tranquada et al., Nature (London) 375, 561 (1995).

[10] B. Lake et al., Science 291, 1759 (2001).

[11] J. Mesot et al. (to be published).

[12] J. Kohlbrecher and W. Wagner, J. Appl. Cryst. 33, 804 (2000).

[13] P. Rasmussen et al. (to be published).

[14] The TSFZ method was used for the crystal growth; see, e.g., T. Nakano, N. Momono, M. Oda, and M. Ido, J. Phys. Soc. Jpn. 67, 2622 (1998).

[15] T. Schneider and J. M. Singer, J. Supercond. 13, 789 (2000).

[16] R. Cubitt et al., Nature (London) 365, 407 (1993).

[17] A. Vostner et al., Solid State Commun. 107, 291 (1998).

[18] B. Keimer et al., Phys. Rev. Lett. 73, 3459 (1994).

[19] E. M. Forgan et al., Phys. Rev. Lett. 75, 1422 (1995); M. B. Walker and T. Timusk, Phys. Rev. B 52, 97 (1995).

[20] L. J. Campbell, M. M. Doria, and V. G. Kogan, Phys. Rev. B 38, 2439 (1988).

[21] D. McK. Paul et al., Phys. Rev. Lett. 80, 1517 (1998).

[22] T. M. Riseman et al., Nature (London) 396, 242 (1998).

[23] A. J. Berlinsky et al., Phys. Rev. Lett. 75, 2200 (1995).

[24] Ji-Hai Xu, Yong Ren, and Ting Chin-Sen, Phys. Rev. B 53, R2991 (1996).

[25] J. Shiraishi, M. Kohmoto, and K. Maki, Phys. Rev. B 59, 4497 (1999).

[26] M. Ichioka, A. Hasegawa, and K. Machida, Phys. Rev. B 59, 8902 (1999).

[27] S. A. Kivelson, E. Fradkin, and V. J. Emery, Nature (London) 393, 550 (1998); A. H. Castro Neto and F. Guinea, Phys. Rev. Lett. 80, 4040 (1998).

[28] H. A. Mook and F. Dogan, Nature (London) 401, 145 (1999). 